\title{
2. Handschriftenreste der Zeit um 1400 in Basel
}

1. Der folgende Abschnitt behandelt nicht ein einzelnes ein- oder mehrblättriges Notenfragment, sondern vier, in unterschiedlichen baslerischen Klosterbeständen überlieferte Manuskriptbruchstücke; ${ }^{1}$ wie es scheint, stehen sie unter sich in zum Teil buchbinderischem und zum Teil inhaltlichem Schreiberzusammenhang. Die folgende Beschreibung der Quellen sowie ihres jeweiligen Inhalts kürzt diese vier Fragmente mit den fetten Buchstaben A-D ab.

Fragmente, Klosterarchiv St. Clara (Corpora 1523-1529, Bd. 1525) [im Folgenden $=\mathbf{A}]$, Basel, Staatsarchiv des Kantons Basel Stadt

Hochformatiges, an allen vier Seiten, aber nur unten mit Textverlust beschnittenes Pergamentblatt von (heute) ca. 30,9 x ca. $32,2 \mathrm{~cm}$, das offenbar als Koperteinband eines Zinsrodels des Basler St. Clara-Klosters von 1525 diente: die Rücken- und Verarbeitungsspuren sind vor allem auf dem recto, das die Außenseite bildete, noch sichtbar. Beide Blattseiten zeigen zwei Spalten von je 11 (noch sichtbaren,) mit in roter Tinte ausgezogenen Notensystemen von ca. 10,5/10 cm (recto, je in beiden Spalten) und ca. 9,8/10,8cm (verso, je in beiden Spalten) Breite. Schwarze Mensuralnotation, rote Initialen für Stimmbezeichnungen, Textanfangswörter, auch rote Pausenstriche. Keine colores.

r: Auf den ersten 6 (linke Spalte) bzw. 7 Notensystemen (rechte Spalte) ein vierstimmiges Kyrie (vgl. Abb. 2a)); die Stimmbezeichnungen „Quadruplum“ und „tenor“ auf der linken, „Triplum“ und „Contratenor“ auf der rechten Spalte. Das erste Kyrie des „Triplum“ ist, um den Vermerk ,primo" ergänzt, erst nach dem Christe und dem zweiten Kyrie dieser Stimme im 4. System der rechten Spalte eingetragen.

Auf den verbleibenden 5 (linke Spalte) bzw. 4 Systemen (rechte Spalte) Beginn eines wohl zum vorangehenden Kyrie gehörend verstandenen

1 Den Hinweis auf die hier behandelten Fragmente A-C und ihre Überlassung zur Veröffentlichung dankt der Autor der großen Freundlichkeit von Herrn Dr. Frank Labhardt, den Hinweis auf Fragment D einer willkommenen Mitteilung von Frau Dr. Veronika Gerz-von Büren (vgl. dazu unten, Anm. 13). 
dreistimmigen Gloria; die Stimmen sind hier nicht mehr wie im Kyrie bezeichnet.

Der C beansprucht die Notensysteme 7-10, Anfang, (linke Spalte) und führt den Text bis „,... propter magnam gloriam tuam“. Im System 10, Mitte, (linke Spalte), beginnt ohne lange Unterbrechung der T; am Ende des Blattes (linke Spalte) bricht sein Text mit ,... glori-“ (aus ,glorificamus te“) $\mathrm{ab}$; die Fortsetzung folgt seitlich verschoben auf dem 11. System (rechte Spalte) und führt, heute nur noch zum Teil lesbar, bis ,... propter magnam gloriam tuam". Das Blatt hat also auf dem recto und, wie sich noch zeigen wird, auf dem verso spaltenweise je 11 Systeme enthalten, und diese sind auf beiden Seiten und in je beiden Spalten fast vollständig erhalten geblieben. Der CT schließlich füllt die Systeme 8-10 (rechte Spalte) des recto; System 11 (rechte Spalte) nimmt Teile des T auf (s. o.).

v (vgl. Abb. 2b)): Die Notensysteme 1-7 (linke und rechte Spalte) bringen, beginnend mit „Domine deus rex celestis ...“, für C und CT Fortsetzung und Ende des auf dem recto begonnenen Gloria. Der T nimmt je die Systeme 8-11 (linke und rechte Spalte) ein; der Spaltenwechsel erfolgt bei ,... peccata mundi/suscipe ...".

K: - (gilt für beide Sätze).

Stilistisch weisen die beiden Ordinariumssätze noch ins 14. Jahrhundert. Das dürfte die weitgehend binäre Mensur beider Sätze nahelegen, aber auch - um einige, freilich allzu scharfe Kategorien, aber doch eine gewisse Anschauung schaffende Begriffe zu gebrauchen - der „motet style“ des Kyrie, das sich zu Beginn des $\mathrm{T}$ an das bekannte chorale Marien-Kyrie (,,cum jubilo“) anlehnt; noch deutlicher wird der Rückweis ins 14. Jahrhundert im Gloria, das fast ganz dem ,simultaneous style“ verpflichtet ist. ${ }^{2}$ Die äußere Form der Notation in zwei senkrechten Parallelspalten - die an ältere Motettenmanuskripte denken läßt - und die im Kyrie verwendeten Stimmbezeichnungen „Quadruplum“ und „Triplum“ mögen diese Datierung zunächst bestätigen; doch ist ungewiß, ob sie auch für den Zeitpunkt der Niederschrift gilt. Er könnte zwar noch vor 1400 gelegen haben, aber vielleicht tut man, im Blick auf das Folgende, besser daran, ihn um $1400 \mathrm{zu}$ vermuten.

2 Vgl. Hanna Stäblein-Harder, Fourteenth-Century Mass Music in France, o.O. 1962 (= Musicological Studies and Documents 7), S. 15-19, bes. S. 16-18. 
Große Fragmente, Klosterarchiv Prediger M 1 (Corpora 1592-1593) [im Folgenden $=\mathbf{B}]$, Basel, Staatsarchiv des Kantons Basel-Stadt

Pergamentdoppelblatt, das 1592, auf dem Kopf stehend, zum Einband eines Corpusbandes des Basler Predigerklosters diente. Es zerfällt in zwei ungleich große Blattanteile: der kleinere mit fol. $<1>$ mißt, seinerzeit am äußeren rechten Rand unter wesentlichem Textverlust beschnitten, ab (altem) Mittelfalz ca. 17,5 x ca. 31,3 cm (Höhe), in dem original erhaltenen umfangreicheren Teil mit fol. $<2>$ ca. $22,4 \mathrm{~cm}$ x ca. $31,3 \mathrm{~cm}$. Zur VergröBerung des beschnittenen fol. $<1>$ auf die ganze, buchbinderisch notwendige Breite ist seitlich an dieses das Pergamentfragment C (s. u.) angeklebt worden, so daß der so hergestellte Koperteinband den neuen PredigerCorpusband umschließen konnte; jener ist auf fol. <2>' auf 1593 datiert.

fol. $<1>$ (vgl. Abb. 2c)) bringt oben eine mutmaßliche Lagen- und Foliobezeichnung: „, $1 \mathrm{w}$ “; offenbar ist damit das erste recto-Blatt einer Lage „W", dann also direkt nach der Lage „V“, gemeint und damit ein Hinweis auf eine einst ziemlich umfangreiche Sammelhandschrift gegeben. Daß so die beiden, durch Gattung und Schreiber sich nahestehenden Agnus-Sätze auf fol. $<1>$ ' und $<2>$ (vgl. Abb. 2d)) auseinandergespreizt worden wären, weil zwischen ihnen weitere, heute verlorene Blätter der Lage „w“ gestanden hätten, dürfte die Deutung des genannten Vermerks nicht erschüttern. - Auf fol. $<1>$ sind 10 Systeme, in der zu unterst stehenden Stimme mit 6 Linien, auf fol. $<1>^{\prime} 9$, auf fol. $<2>7$ und auf fol. $<2>^{\prime} 9$ Systeme durchgezogen worden; auf fol. $\langle 1\rangle$ und $\langle 2\rangle^{\prime}$ sind sie teilweise oder durchgehend sechs-, auf fol. $\langle 1\rangle^{\prime}$ und $<2>$ fünflinig. Schwarze Mensuralnotation; keine colores, einzig auf fol. $<1>$ im „Trůmpet“ weiße colores. Fol. $<1>$ ' und $<2>$ sind von unbekannter Hand je mit dickem Rötelstrich diagonal durchgestrichen worden.

fol. $<1>$ (vgl. Abb. 2c)): „O regina <saba?> grata salomonis mira data ...“ (Zeilenreim ,...ata“): Motette mit den Stimmen <Cantus>, „Tenor", "Contratenor" und "Trůmpet ... concordans absque contratenore“. Die Suche nach einer Parallelüberlieferung des im C gegebenen Oberstimmentextes - der, wenn die ergänzende Wortkonjektur im Incipit zutrifft, biblisch möglich und dem Metrum entsprechend Königin von Saba und König Salomon verbindet - ist erfolglos geblieben. Das Stück scheint, soweit sein beschädigter Zustand eine Beurteilung zuläßt, nicht aus deutschsprachigem oder zentraleuropäischem Gebiet zu stammen: wie fast alle eine „Trumpet“[-(bzw.)],,Trompetta“-Stimme vorsehenden Werke dürfte die kompositorisch anspruchsvolle Motette von einem französischen Komponisten aus den ersten Jahrzehnten des 15. Jahrhunderts stammen; 
nur der fragliche Stimmenvermerk geht wohl auf einen Schreiber deutscher Zunge zurück. Unter dieser Voraussetzung ist dann die Stimmbezeichnung „Trůmpet“ sogleich auffällig. Margret Bent hat unlängst Klärendes zu den „Trompetta“- und „Concordans"-Stimmen vorgetragen: ${ }^{3}$ danach haben von dem Bestand von zwölf nachgewiesenen „Trompetta“-Kompositionen immerhin acht eine solche Stimme als nachträgliche Zugabe zu einem in ihnen bereits vorhandenen und selbst-konsistenten Duett meist mittlerer Stimmen nachgetragen erhalten. Diese sind dann oft T/CT-Parte. Die Stimmbezeichnungen "Trompetta“, "Contratenor" und „Concordans" werden hierbei gerne untereinander in direkte Beziehung gesetzt, ja in Konkordanzhandschriften sogar für die gleiche Stimme verschieden angewandt. Das vorliegende Stück, für die weitere Diskussion dieser Fragen nicht unwichtig, scheint den Bent'schen Einsichten zu entsprechen: die Stimmmarke „Trůmpet“ zeigt sich im Basler Fragment graphisch etwas anders ausgeführt als diejenigen für die, den textierten $\mathrm{C}$ tragenden textlosen Parte der als Paar erscheinenden T- und CT-Stimmen. Die Tatsache, daß der Schreiber nach der „Trůmpet“-Marke die Angabe „,concordans absque contratenore" beifügt, bedeutet offenbar, daß in diesem Fall jenes „Trůmpet“, anderswo auch als „,concordans“ bezeichnet, den vorhandenen „Contratenor" ersetzen soll, ein Vorgang, der zwar das gegebene Duett der Mittelstimmen aufhebt, aber umso näher liegt, als schon der CT erkennbar im Tonraum springt, wenn auch nicht so weit und fanfarenhaft wie das „Trůmpet“.

$\mathrm{K}:-$

fol. $<1>^{\prime}$ (vgl. Abb. 2d)): „Angnus dei“, 3v., bei geringem Textverlust am linken Rand; eine musikalische Vorlagemelodie oder -komposition hat sich nicht finden lassen. ${ }^{4}$

3 Vgl. Margaret Bent, Trompetta and Concordans Parts in the Early Fifteenth Century, in: Music as Social and Cultural Practice. Essays in honour of Reinhard Strohm, Woodbridge 2007, S. 38-73. Vielleicht verdient schon hier die Tatsache ausdrückliche Beachtung, daß das noch zu erörternde Fragment Zwettl, wohl etwas später als B entstanden, aber ebenfalls „Trompetta“-Bezogenes enthaltend, offenbar in Basel entstanden ist; diese Provenienz ist durch Peter Wrights Identifikation der Schreiberhand des Johannes Lupi in diesem Fragment glaubwürdig vertreten worden, für den auch Abschrift von Teilen der Trienter Codices festgestellt ist; vgl. Peter Wright, The compilation of Trent 87-I and 92-II, in: Early Music History 2 (1982), S. 237-271, sowie ders., On the origins of Trent 87-I and 92-II, ebda. 6 (1986), S. 245-270.

4 Die Durchsicht von Martin Schildbach, Das einstimmige Agnus und seine handschriftliche Überlieferung vom 10. bis zum 16. Jahrhundert, Diss. Erlangen-Nürnberg 1967, blieb erfolglos. 
$\mathrm{K}:-$

fol. $<2>$ (vgl. Abb. 2d)): „Angnus dei“, 3v. (dabei Agnus III = Agnus I); eine musikalische Vorlagemelodie oder-komposition hat sich nicht finden lassen. ${ }^{5}$

$\mathrm{K}:-$

fol. $<2>^{\prime}$ (vgl. Abb. 2c)): „Et in terra pax“, 1v., am Ende auch mehrstimmig. Obschon vor dem „Amen“ ein Mensurzeichen steht, scheint davor eine grundsätzlich einstimmig-chorale Gloria-Melodie mit mensural organisierter „Cantus-fractus“-Rhythmik notiert zu sein: dafür spricht vor allem die Tatsache, daß sie aus durchweg geradzahligen Notenwerten gebaut ist und keinerlei Pausen vorsieht; sie hat sich in Gradualien oder Kyrialien nicht wiederfinden lassen, ${ }^{6}$ weil sie wohl eine spätmittelalterliche Neukomposition ist, aber auch, weil in dieser Zeit einstimmige "Cantus-fractus“typische Ordinariumssätze nicht zum Gloria, sondern wohl ausschließlich zum Credo cardinale geschaffen wurden - insofern ist der vorliegende „Cantus-fractus“-Beleg zum Gloria exzeptionell. Gegen diese Identifikation spricht auch nicht das Auftreten mehrere kleinwertiger Unterterzklauseln, wie man sie, wenngleich irrig, vor allem in mehrstimmigen Sätzen erwarten würde. ${ }^{7}$

$\mathrm{K}:-$

Darunter und ebenfalls auf dem Kopf stehend Titel des mit diesem Pergamentmaterial eingebundenen neuen „Corpüsbüch von Johan. $<$ nis $>A^{\circ} 92$ | Biß wiederumb johann anno 1593“, darunter in weißer Mensuralnotation wohl auch erst des spätesten 16. Jahrhunderts Federproben zum Text „Gloria tibi dominj [sic] ... L: D: S:“.

Das fragliche Doppelblatt überrascht durch die äußere Vielgestaltigkeit seiner Eintragungen: Ordinariumssätze, sowohl einstimmig als auch durchgehalten mehrstimmig, eine Motette mit, wie sich zeigen wird, damals ,"moderner" Stimmenfunktion; mit dieser stilistischen Verschiedenheit hängt auch die Beurteilung ihrer Entstehungszeit zusammen. Vielleicht ist das Gloria auf fol. $<2>^{\prime}$ in seiner "Cantus-fractus“-Nähe - nachdem diese be-

5 Die Recherchen bei Schildbach, ebda., führten auch hier zu keinem Ergebnis.

6 Gleiches wie für Anm. 4 und 5 (s. oben) gilt auch für die Recherchen nach dieser Melodie bei Detlev Bosse, Untersuchung einstimmiger mittelalterlicher Melodien zum „, Gloria in excelsis Deo“, Diss. Erlangen 1955.

7 Vgl. Marco Gozzi/Francesco Luisi, Il canto fratto - L'altro Gregoriano, Rom 2005 (= Miscellanea Musicologica 7), S. 15ff. (M. Gozzi), und passim. 
sondere rhythmische Gestaltung bereits früh auftritt ${ }^{8}$ - das älteste und noch ins 14. Jahrhundert zurückreichende Stück auf B. Die Motette O regina auf fol. $<1>$ ist jedoch mit ziemlicher Gewißheit eine Schöpfung des ersten Drittels des 15. Jahrhunderts. Darauf deutet die weiße Notation der colores, wohl auch in der wie hier räumlich engen Kombination mit Proportionsangaben; entscheidend für eine noch etwas genauere Datierung der Komposition und vermutlich auch ihrer Niederschrift auf die Jahre etwa von 1420 bis nach 1430 ist jedoch die Zugehörigkeit des Satzes zu jener „Trompetta"-Mode, die in eben dieser Zeit eine gewisse Blüte erlebt; die Beziehungen von „Trůmpet“-, „Contratenor“ und „Concordans“-Bezeichnungen untereinander sowie die satztechnischen Funktionen dieser Stimmen sind schon oben berührt worden. ${ }^{9}$ Die Angabe „Trůmpet“ scheint, wie schon vermutet, auf einen deutschsprachigen Schreiber zu deuten. Schließlich bleiben die beiden Agnus-Sätze, bei denen besonders die etwas einfache Faktur des zweiten (fol. $<2>$ ) auffällt, indem dort Agnus I und III in allen drei Stimmen so gut wie identisch, aber trotzdem ausgeschrieben sind. Die beiden Agnus-Sätze sind nicht nur vom gleichen Schreiber eingetragen, sondern könnten auch vom gleichen Komponisten stammen. Man möchte sie ins frühere 15. Jahrhundert datieren.

Große Fragmente, Klosterarchiv Prediger M 1 (Corpora 1592-1593) [im Folgenden $=\mathbf{C}]$, Basel, Staatsarchiv des Kantons Basel-Stadt

(Heute) hochformatiges Pergamentfragment, auf einer Seite unter erheblichem Textverlust beschnitten, heute ca. 30,9 × $12,3 \mathrm{~cm}$ messend; das Fragment wurde außen rechts an das seinerseits beschnittene eine Blatt (fol. $<1>$ ) von $\mathbf{B}$ angeklebt, um einen volldeckenden Koperteinband zu ermöglichen (s. oben); dabei kam das recto von $\mathbf{C}$ auch seinerseits in rectoPosition unter den Rand von fol. $<1>$ von $\mathbf{B}$ zu liegen. - Das recto von $\mathbf{C}$ (vgl. Abb. 2e)) zeigt 11, das verso (vgl. Abb. 2f)) 6 Systeme zu 5 Zeilen; keine colores. Es sind auf recto und verso zwei neue und je verschiedene Schreiber vertreten.

r: Fragment des $\mathrm{C}$ und des $\mathrm{T}$ des gut bezeugten Ars-nova-Virelai Par maintes foys, 3v. von Johannes Vaillant (vgl. Abb. 2e)). Erhalten sind die

8 „Cantus-fractus“-Rhythmik im choralen Repertoire ist seit der Wende vom 13./14. Jahrhundert nachgewiesen, vgl. Gozzi, ebda., S. 7.

9 S. oben, zu fol. <1>. - Vgl. dazu auch Margaret Bent, Naming of Parts: Notes on the Contratenor, c. 1350-1450, in: Uno gentile et subtile ingenio. Studies in Renaissance Music in Honour of Bonnie J. Blackburn. Ed. by M. Jennifer Bloxam, Goia Filocamo, and Leofranc Holford-Strevens, < Turnhout> 2009, S. 1-12. 
Partien des C, 58,9-65,3; 70,4-76,5; 81,1-Schluß (= clos), sowie des T, 65,2-Schluß (= clos). ${ }^{10}$

K: - Bruxelles, Ste. Gudule/Mons, Leclercq; anon.

- Cambrai 1328, fol. 20 (Fragment); anon.

- Chantilly 564, fol. 60, ,Jo. Vaillant“"

- Grottaferrata 197, fol. 12'-13 (Fragment); anon.

- Innsbruck, Wolkenstein B, fol. 22'-23 (Kf.); anon.

- Lucca 184, fol. 74'-75'; anon.

- München 14274, fol. 27'-28 (Kf.); anon.

- Straßburg 22, Nr. 101, ,Wilhelmi de Maschaudio“" (Kf.)

- Wien, Wolkenstein A, fol. 19'-20 (Kf.); anon.

- Warszawa 61, 1, fol. 278-287; ,,per mantes phorys“ (Traktatzitat); anon.

- Warszawa 61, 2, fol. 288-293; ,per montes foijs“ (Traktatzitat); anon.

- Wrocław 16, fol. 144'-152'; „Par mantes foys“ (2 Traktatzitate) anon.

v: „exultet vena mea [irrig statt „,mea vena“] quodlibet exphylomeua [sic] ...“" (Zeilenreim ,...ena“): Oberstimme einer dreistimmigen Motette (vgl. Abb. 2f)). Noten sind nur in den ersten vier Systemen niedergeschrieben, der Text jedoch ist bis zum unteren Blattende vornotiert; die Unterstimmen sind auf dem voll ausgenutzten Raum nicht erkennbar, so daß man annehmen muß, daß die Niederschrift der Komposition, wenn sie überhaupt noch komplettiert worden wäre, auf dem folgenden recto gestanden hätte. Die Tatsache, daß die Eintragung dieses Stücks auch in der Straßburger Quelle (s. unten) einstimmig war und die Schlüsselung sowie die Incipits hier und dort identisch waren, schließlich auch die Einsicht, daß die zwei allein in der Leidener Konkordanz erhaltenen Unterstimmen (s. unten), wenngleich sehr schwer lesbar, musikalisch zum Basler/Straßburger C-Incipit passen, ${ }^{11}$ bestätigen, daß die Motette mehrstimmig war.

K: - Leiden 342 (Fragment); anon.

- Straßburg 22, Nr. 42; anon.

Der Worttext dieses „Motetus Philomena“, wie das Stück in Straßburg 22 benannt war, ist in $\mathbf{C}$ verhältnismäßig am besten überliefert. Freilich ist er

10 In neuerer Zeit ist der Satz ediert in Polyphonic Music of the Fourteenth Century... (= French Secular Music, vol. 19, ed. Gordon K. Greene), Monaco 1982, Nr. 100, S. 170-174, sowie S. 195f., Nr. 100.

11 Vgl. Lorenz Welker, Musik am Oberrhein im späten Mittelalter. Die Handschrift Strasbourg, olim Bibliothèque de la Ville, C. 22, Hab.schr. (maschr.) Basel 1993, Bd. 2, Inventar S. 14, zu Nr. 41/42. Herrn Kollegen Lorenz Welker, München, sei für die Überlassung eines Exemplars seiner Arbeit bestens gedankt. 
infolge der Beschneidung des rechten Blattrandes wohl etwa zur Hälfte und am Ende ganz verloren und im vorhandenen Teil mitunter schwer lesbar. Nachdem van den Borren aufgrund jenes Straßburger Titels „Motetus Philomena“ und, ebenda, des Incipits „Exultet mea vena quodlibet ex Phylomena" [sic] (so im Prinzip auch in $\mathbf{C}$ ) in der vorliegenden Motette ein frühes quodlibet-artiges Werk vermutet und in der Musikernamen-Reihung im Text der etwa gleichzeitigen Motette „Apollinis exclipsatur“ (ebenfalls in Straßburg 22, Nr. 100) tatsächlich den Namen eines "Jugis Philomena“ (also, metaphorisch, einer „unabläßig singenden Nachtigal“) gefunden hat, ${ }^{12}$ ließe sich die sonst unverständliche Form der noch vorhandenen, aber inhaltlich nicht verbindbaren Reste des fraglichen Oberstimmentextes etwas besser begreifen: Es scheint in ihnen eine quodlibet-artige Reihung verschiedener und umständlich-gelehrt formulierter Einzelaussagen vorzuliegen, die allerdings kaum in einen inhaltlichen Zusammenhang zu bringen sind. Die einzige erkennbare, aber eher formale Ordnung erscheint in den die ursprünglichen Textzeilen je abschließenden Reimwörtern auf „...ena“, also etwa „habena“ (Zügel), „foena“ (Hafer), „cathena“ (Kette, Fessel), „mala ... dena“ (zehn Übel), ,,venena“ (Gift), ,avena“ (Hafer), „,balena“" (Walfisch), „epystena“ [sic] Grabstein) u.a. Um in diesem durch Verlust und Zerstörung beeinträchtigten, überdies anspruchsvoll und metaphern-reich formulierten Text einen auch nur halbwegs überzeugenden Sinn (wenn es denn überhaupt einen gegeben hat) zu erkennen, müßte man erst über eine wirklich zuverläßige neue Konkordanz verfügen können.

Stärker als die übrigen Blätter macht $\mathbf{C}$ den Eindruck nicht nur von bunt kopiertem „Sammelgut“, sondern auch von erheblicher Vorläufigkeit und Unbestimmtheit des Notierten. Das liegt weniger an der nicht eben überzeugend präsentierenden Eintragung auf dem recto, deren Schreiber die Musik schlecht disponiert ganz in die obere Seitenhälfte gedrängt hat; aussagekräftiger ist vielmehr, daß hier die Notensysteme ebenfalls nur bis zur Blattmitte, also wohl spontan nur gerade für diese Eintragung gezogen worden sind. Das deutet an dieser Stelle eher auf einen „Füller“ von leerem Raum als auf die geplante Notation eines bewußt bereitgestellten Vorlagenstücks. In die gleiche Richtung des Vorläufigen deutet das verso, das nur eine unfertig gelassene Eintragung enthält, übrigens diejenige eines wohl noch tiefer ins 14. Jahrhundert gehörenden Stückes.

12 Vgl. Charles van den Borren, Le Manuscrit Musical M. 222 C. 22 de la Bibliothèque de Strasbourg ( $X V^{e}$ siècle) brulé en 1870, et reconstitué d'après une copie partielle d'Edmond de Coussemaker, Anvers 1924, S. 75f., zu Nr. 42, sowie S. 113, zu Nr. 100. 
Über die Frage, ob das Blatt je in einen größeren Manuskriptzusammenhang gehört habe, ist ein klares Urteil unmöglich; es wäre zwar eine gesonderte und zugefügte Musikniederschrift an einer leeren Stelle irgendeines Textmanuskripts denkbar, doch wird man auch die Vermutung nicht los, daß - was schon die Konkordanzen nahelegen das verbrannte Manuskript Straßburg 22 vom Jahre 1411 nach Inhalt und Äußerem an gewissen Stellen etwa so bunt gemischt ausgesehen haben könnte wie $\mathbf{C}$.

Basel, Staatsarchiv des Kantons Basel-Stadt, Fragmente Klosterarchiv St. Clara Q 1, $1522[$ im Folgenden $=\mathbf{D}]$

Einzelnes Pergamentblatt des frühen 15., in Teilen vielleicht noch des späten 14. Jahrhunderts; $37,2 \times 24,5 \mathrm{~cm}$ (Hochformat); eine Längsseite ist etwas beschnitten. 1522 wurde das Fragment als Deckblatt zu einem Rechnungsrodel des Basler Clarissenklosters verwendet. Die bei diesem Deckblatt äußere (recto-)Seite (vgl. Abb. 2g)) ist stark abgerieben und verblaßt, aber in Ultraviolett-Photographie so weit lesbar, daß wenigstens der ganze Notentext, der Worttext jedoch nur teilweise übertragen werden kann; die innere (verso-)Seite (vgl. Abb. 2h)) ist nur an der Schnittkante leicht verwischt. ${ }^{13}$ Auf beiden Seiten sind je zwei parallele senkrechte Spalten von je 11 in roter Farbe rastrierten Notensystemen ausgezogen; da eine Folio-Zahl nirgends zu erkennen ist, läßt sich über den Umfang der Provenienzhandschrift, aus der das Blatt ausgeschnitten wurde, zunächst nichts sagen.

r (vgl. Abb. 2g)), Spalte links: dreistimmige, nicht-isorhythmische Motette mäßigen Umfangs; die Texte aller drei Stimmen sind nur schwer lesbar (leider schon ihre Anfänge nicht), doch verraten die gesicherten C-Zeilen „... ut filias Clare nemini detur assimulare ...“ und „,... Jpsis [sc. filiis Clare]

13 Der Autor hat das Blatt bereits 1978 in Neue Quellen zur mehrstimmigen Musik des 15. und 16. Jahrhunderts in der Schweiz, in: Schweizer Beiträge zur Musikwissenschaft 2 (1978), S. 57-83, bes. S. 57-59, veröffentlicht, freilich noch ohne Kenntnis der Fragmente A-C und mit photographischer Abbildung allerdings nur der gut erhaltenen $\mathbf{v - S e i t e . ~ N a c h d e m ~ d a s ~ B l a t t ~ a b e r ~ h i e r ~ i n ~ e i n e n ~ F r a g m e n t e n - ~}$ Komplex eingefügt erscheint, ist es der Vollständigkeit halber nötig, D ganz, also auch mit der r-Seite, zu reproduzieren. Auch an dieser Stelle sei der Photoabteilung der Kriminaltechnischen Abteilung der Staatsanwaltschaft Basel-Stadt mit den Herren Claude Flückiger, Daniel Buchser, Pascal Müller und Fabian Corbaz sehr angelegentlich gedankt, die von der abgeriebenen r-Seite eine UltraviolettReproduktion hergestellt und damit die Lesbarkeit der Niederschrift gegenüber einer früheren Fluoreszenzaufnahme wesentlich verbessert haben (vgl. Abb. 2g)). In der Quellenbeschreibung von D, v, Spalte rechts, sind, aufgrund weiterer Beobachtungen, gegenüber 1978 einige Modifikationen vollzogen. 
hoc carmen offero pro munere ..." (Zeilenreim ,...are“), daß es sich um eine einem Clarissen-Kloster, dann sehr wahrscheinlich demjenigen im rechtsrheinischen Basel zugedachte und deswegen die älteste mit Basel ${ }^{14}$ verbundene mehrstimmige Komposition handelt.

$\mathrm{K}:-$

r, Spalte rechts: da sich keine Textwiederholung feststellen läßt, liegt eine wohl nur einstimmige Marien-Cantio vor (System 4: ,...Gaude [?] plaude ac ympnusa symphonisa discantisa dic christi [?] ... digne: Veni dulcis consolatrix veni uite reparatrix ..."), mit nur teilweise lesbarem, wohl rhythmischem Marien-Text und schwer lesbarem Notentext, insgesamt vermutlich von einer anderen Hand als derjenigen der vorher besprochenen Eintragung. Unter dem letzten System sind von einer etwas späteren Hand Ansätze zu einem deutschen Liebesliedtext nachgetragen:

„Jch kam an [?] < ei $>$ ne liebesthur | die thur die was beschlossen || der Rigel vnd der <? $>$ fur [?] |

Jch was kein [?] grosser liden | dann so ich dich herz lieb můß miden“"

Dieser Liedtext ist auch sonst und in vollständigerer Form bekannt, wenngleich mit mehreren Textvarianten. ${ }^{15}$

$\mathrm{K}:-$

v (vgl. Abb. 2h)), Spalte links: Virelai „Je languis damors“, 2v.. Dem Schriftductus und der korrekten Textschreibung nach wohl von einem Franzosen stammende oder in frankreichnahem Gebiet entstandene Notation des frühen 15., vielleicht noch des späten 14. Jahrhunderts. Als (nur) zweistimmige Version und nach vergleichender Überprüfung der musikalischen Lesarten dürfte die vorliegende Fassung der Konkordanz in Prag 9 (s. oben) verhältnismäßig nahestehen, und damit, in lehrreicher

14 Um der Klarheit willen sei ergänzt, daß es sich beim „,Kloster St. Clara“ im vorliegenden Text durchweg um das rechtsrheinische Stift dieses Namens (auf Kleinbasler Boden) und nicht um das linksrheinische „Clarissenkloster in Gnadental“ (auf Großbasler Boden) handelt.

15 Vgl. Joachim Lüdtke, Die Lautenbücher Philipp Hainhofers (1578-1647), Göttingen 1999 (= Abhandlungen zur Musikgeschichte, Bd. 5), S. 188-191, bes. S. 188, zu „Ich gieng. bey eitler nacht" bzw. „Ich ging wohl bei der Nacht“ (älteste bisher bekannte Überlieferung um 1478 im Rostocker Liederbuch) sowie S.275 (mit Konkordanzen). Herrn Dr. Joachim Lüdtke (Fürth) sei auch hier für den Hinweis auf dieses Lied, seinen Text und weitere Informationen bestens gedankt. Die Wiedergabe der beiden Textanfänge sucht mutmaßliche Enden ( || ) und Binnenreime ( | ) der Zeilen anzuzeigen. 
Weise, wiederum einer Handschrift, die oberrheinischer Herkunft ist (wohl Straßburg?) und ebenfalls Beziehungen zum Clarissenorden erkennen läßt. ${ }^{16}$

K: - Florenz 26, Nr. 128, 3v.; anon

- Paris 568, fol. 132'-133, 3v.; anon.

- Paris 6771, Nr. 147, 4v.; anon.

- Parma 75, fol. 2', 3v.; anon.

- Prag 9, Nr. 12, 2v.; anon.

- Straßburg 22, Nr. 106, 3v.; anon.

- Augsburg 38, fol. 173'-180 / Philadelphia 36, fol. 207-216’' ,je languis" (Traktatzitat); anon.

- Melk 950, fol. 76 (188)'-83 (204)’; „Yelangis“ (Traktatzitat); anon.

- Sterzing, fol. 1-3; ,Jelangwis“" (Traktatzitat); anon.

- Warszawa 61, 1, fol. 278-287; ,,zelangwis“ (Traktatzitat); anon.

v, Spalte rechts: Weihnachts-Cantio „Ab archanis [sic] oritur“, 3v. (, ... plaudite iuvencule ob natale parfuli ..."), gegenüber vorher von einer anderen, wohl deutschen Hand etwa des frühen zweiten Drittels des 15. Jahrhunderts notiert. Die feminine Wortendung ,iuvencule“ sollte nicht dazu verführen, das Stück ebenfalls auf die Clarissen (s. oben, r, Spalte links) zu beziehen; es folgen jenen im Text nämlich sogleich die maskulinen „parvuli“, so daß hier doch einfach sowohl Mädchen als auch Knaben angesprochen sind. Nachdem die im Folgenden aufgeführten Konkordanzen beide böhmischer Herkunft sind, liegt hier wohl ein weiterer Beleg für die auffällige, wohl schon durch die Zerstörungen der Hussitenkriege in Böhmen selber verursachte Erscheinung vor, daß vereinzelte Fassungen von dort entstandenen Cantionen mit zeitlichem Vorsprung in ,westdeutschem“ Bereich auftreten, vor einer - weitgehend verlorenen - Hauptüberlieferung in Böhmen selber. ${ }^{17}$ Gegenüber der Konkordanzüberlieferung findet sich hier ein CT zugefügt.

16 Am Anfang des überhaupt mit Straßburg verbundenen Manuskripts steht ein Pergamentkalendarium aus einem Straßburger Clarissenkloster; vgl. Friedrich Kammerer, Die Musikstücke des Prager Kodex XI E 9, Augsburg 1931 (= Veröffentlichungen des Musikwissenschaftlichen Instituts der Deutschen Universität in Prag, Bd. 1), S. 11.

17 Vgl. auch Kurt von Fischer, Zum Repertoire der böhmischen Mehrstimmigkeit vom 14. bis zum 16. Jahrhundert - Die Verbreitung des böhmischen Repertoires außerhalb Böhmens, in: Colloquium Musica bohemica et europaea Brno 1970, Brno 1972, S. 5561, bes. S. 59-61. Unbekannte neuere Belege für diese besondere Quellenlage, teilweise mit Stücken von Petrus Wilhelmi, bieten Tom R. Ward, Music in the University: The Manuscript Leipzig, Universitätsbibliothek, MS 1084, in: Gestalt und Entstehung musikalischer Quellen im 15. und 15. Jahrhundert. Hrsg. von Martin 
K: - Chrudim, Nr. 15, 2v.; anon.

- Hradec Kralové 6, Nr. 28, 2v.; anon.

2. Es soll im Folgenden versucht werden, die vorgefuihrten Fragmente und ihre Übereinstimmungen in Quellengestalt und Schreiberhänden sowie im Inhaltlichen des beschriebenen Fragmentenkomplexes, soweit möglich, zu kommentieren.

A, geschrieben wohl um 1400, vollzieht, was damals eigentlich nicht mehr zeitgemäß ist, die Niederschrift der Ordinariumssätze in Spalten. Allerdings braucht man das - trotz den zeitlich ebenfalls zurückweisenden, aber auch sonst, etwa noch in Straßburg 22 bezeugten Stimmbezeichnungen „Quadruplum“ und „Triplum“ - dann nicht mehr für ein deutlich rückwärtsgerichtetes Charakteristikum zu halten, wenn man sich daran erinnert, daß $\mathbf{D}$ beiderseits ebenfalls in Spalten notiert ist. Ein genauer Vergleich von $\mathbf{A}$ und $\mathbf{D}$ ergibt darüberhinaus, daß bei diesen Blättern die Rastrierungen nach Zahl der Systeme und deren Detailabmessungen (der Abstand der beiden obersten Systemlinien neigt zu etwas mehr Weite als sonst) so ähnlich, ja gar übereinstimmend sind, daß man schließen darf, daß die beiden Blätter derselben Provenienzhandschrift entstammen, ein Umstand, der auch deshalb glaubhaft wird, weil die beiden Fragmente 1522 bzw. nach 1525 in derselben Buchbinderwerkstatt, nämlich der für das Stift St. Clara in Basel tätigen oder gar, wenn vorhanden, stiftseigenen Werkstatt, zur Einfassung der genannten Kloster-Corpora verwendet worden sind. Zugleich scheint der genannte Umstand anzudeuten, daß noch nach 1525 Makulaturpergament aus einer Sammelhandschrift vorrätig war, die schon drei Jahre zuvor Material zu diesem Zweck geliefert hatte, folglich ihrer originalen Gestalt nach einen beträchtlichen Umfang gehabt haben dürfte.

Eine Beziehung zwischen einzelnen der vier Fragmente scheint sich allerdings auch dann zu eröffnen, wenn man speziell der Schrift eines der hier beteiligten Kopisten nachfragt. Denn es handelt sich offenbar um den gleichen Schreiber, der auf $\mathbf{B}$, fol. $\langle 1\rangle^{\prime}$ und $<2>$, die beiden Agnus-Sätze und auf $\mathbf{D}$, verso, rechte Spalte, die Cantio „Ab archanis oritur" notiert hat: deutlicher als in den Musiknoten gibt sich diese Übereinstimmung in der Textschrift zu erkennen, etwa in der Bemühung des Kopisten um eine Verzierung des Anfangsbuchstabens „A“ oder in den beiderseitigen Stimmbezeichnungen „Contratenor“" oder „Tenor“; auch die C-Schlüssel und die dreifachen Schlußstriche weisen in diese Richtung. Damit schließen sich auch die im Prediger-Kloster und die für St. Clara vermakulierten Frag-

Staehelin, Wiesbaden 1998, S.21-34 (Leipzig), sowie Joachim Lüdtke, KÜ IV, S. 436-441 mit Abb. 3a)-d) (Lüneburg) und ders., KÜ VI, S. 211-219 mit Abb. 2a)c) (Frankfurt/M.). 
mente untereinander enger zusammen. Man neigt danach zur Annahme, daß dieser Schreiber in Basel selbst gewirkt und damit die Spuren seines Schaffens an verschiedenen Orten der Stadt, also in Manuskripten, die damals ins Prediger- und ins Clarissen-Kloster gelangten und buchbinderisch verarbeitet wurden, hinterlassen habe. - Eine zunächst ebenfalls vermutete Identität der Kopisten von $\mathbf{C}$, recto, und D, recto, linke oder rechte Spalte, scheint nach genauer Prüfung nicht gegeben.

3. Welche Folgerungen ergeben sich aus dem vorstehend Ausgeführten? Zunächst lassen sich die Fragmente A-D in zwei Provenienzgruppen ordnen. Die eine Gruppe besteht aus den Blättern $\mathbf{A}$ und $\mathbf{D}$, die nach äußerer Gestalt, Verarbeitungsprovenienz und -alter als zusammengehörig erscheinen und offenbar aus der gleichen Ursprungshandschrift des 15., vielleicht sogar noch des 14. Jahrhunderts stammen. Dieses Manuskript war in Spalten angelegt und, wie man schließen muß, ursprünglich nur in Teilen beschrieben; die Vielfalt der in $\mathbf{A}$ und $\mathbf{D}$ enthaltenen Stücke und die verschiedenen beteiligten Kopistenhände machen den Sammelcharakter der Handschrift deutlich. Die sich über zumindest drei Jahre erstreckende Dauer der buchbinderischen Verarbeitung dieses Manuskripts in den zwanziger Jahren des 16. Jahrhunderts legt überdies die Vermutung nahe, daß jenes eine verhältnismäßig umfangreiche Handschrift war; die an die Clarissen adressierte Motette auf $\mathbf{D}$, recto, linke Spalte, und die spezifische Überlieferungsnähe des Virelai ,Je languis damors“ auf dem verso, linke Spalte, zu der auch mit einem Straßburger Clarissen-Kloster verbundenen Handschrift Prag 9 (s. oben) lassen vermuten, daß die Bindung von $\mathbf{A}$ und $\mathbf{D}$ an das Basler Kloster St. Clara nicht erst im 16. Jahrhundert, sondern schon viel früher erfolgte. $\mathrm{Ob}$ das gesamte Ursprungsmanuskript schon in direkter Nähe zu St. Clara in Basel entstand, ${ }^{18}$ bleibt jedoch offen.

Die zweite Fragmenten-Gruppe verbindet zunächst B und C miteinander, dies allerdings nur durch die äußerliche, etwa gleichzeitig und am gleichen Ort erfolgte buchbinderische Verarbeitung. Ein Entstehungszusammenhang der Ursprungshandschriften von $\mathbf{B}$ und $\mathbf{C}$ ist mit einer überein-

18 Wenn das zutreffen sollte, dürfte man sich sogar ausmalen, die Basler Clarissen selber hätten einzelne, vermutlich einfachere mehrstimmige Stücke aus den hier behandelten Fragmenten gesungen. Mehrstimmig singende Clarissen sind, wenn auch nicht in Basel, bezeugt; vgl. z. B. für das Kärntner Stift Bischoflack im Jahr 1486: Helmut Federhofer, Beiträge zur älteren Musikgeschichte Kärntens, Carinthia I, Bd. 145 (1955), S. 372-409, bes. S. 375, oder für das Freiburger Franziskanerinnenkloster St. Clara in den Jahren 1556-1598 (anfänglich unter musikalischer Anleitung Glareans): Antonia E. Harter-Böhm, Zur Musikgeschichte der Stadt Freiburg im Breisgau um 1500, Freiburg/Br. 1968 (= Veröffentlichungen aus dem Archiv der Stadt Freiburg im Breisgau 10), S. 46f. 
stimmenden Manuskript-Gestalt oder gleichen Schreibern nicht zu begründen, ja wegen des älteren „Exultet ...“-Restes auf $\mathbf{C}$, verso, vielleicht nicht einmal wahrscheinlich. Zumindest B könnte aber in Basel selbst entstanden sein, nicht nur weil das ebenfalls aus Basel stammende „Trompettafreundliche" Fragment Zwettl, mit $\mathbf{B}$ übereinstimmend, die spezifische Stimmbezeichnung „Concordans“ wie in der Motette „O regina“, in $\mathbf{B}$, fol. $<1>$, verwendet; vielmehr und vor allem erscheint in $\mathbf{B}$, auf fol. $<1>$ ' und $<2>$ dieselbe Schreiberhand, die auch in $\mathbf{D}$, verso, rechte Spalte, tätig gewesen ist. Man wird nun nicht vorschnell so weit gehen wollen, A, B und $\mathbf{D}$ als aus derselben Provenienzhandschrift stammend auszugeben dagegegen spricht schon das Blatt-Format von B. Aber der Schluß darauf wird erlaubt sein, daß hier Reste von zwei umfangreichen, vielleicht sogar drei Basler Handschriften mit mehrstimmiger Musik greifbar werden, die aus dem späten 14. und dem frühen 15. Jahrhundert stammen und die Arbeitsspuren eines oder mehrerer in Basel tätiger Kopisten zeigen; daß auch B ein umfangreiches Provenienzmanuskript bezeugt, ergibt sich aus der oben erwähnten Notiz, die über zwanzig Lagen vermuten läßt.

Mit der Musikübung des Basler Konzils möchte man aus chronologischen Gründen keines der Blätter in Zusammenhang bringen; ihre Eintragungen dürften fast alle älter sein. Näher liegt es jedoch, an irgendwelche Sammelhandschriften etwa jener Art zu denken, wie sie in Straßburg 22 belegt ist; dies gilt umso mehr, als sich zum einen die Überlieferungsnähe gerade zu Straßburg an verschiedenen Stellen der vorstehenden Ausführungen angedeutet hat, zum anderen Straßburg 22 nach neueren Forschungen einige Jahre nach seiner Entstehung im aargauischen Zofingen, 1411, wohl ebenfalls nach Basel gelangt ist ${ }^{19}$ und überdies beide Kompositionen von $\mathbf{C}$

19 Dieser Schluß ergibt sich daraus, daß die auf 1442 datierte Eintragung zweier umfangreicher und weitgehend übereinstimmender Musiktraktate aus Straßburg 22 auch in der Basler Handschrift F. VIII. 16 von dem damals in Basel anwesenden Predigermönch Albrecht Löffler vorgenommen worden ist, folglich dort noch als Vorlage zur Verfügung stand; vgl. Martin Staehelin, Bemerkungen zum verbrannten Manuskript Straßburg M.222 C.22, Mf 42 (1989), S. 2-20, bes. S. 5-12 und 15-20. Daß Lorenz Welker, Untersuchungen zu den Traktaten der Handschrift Strasbourg, Bibliothèque Municipale 222 C. 22, Lic.arbeit (maschr.) Zürich 1989, z. B. S. 76, darauf insistiert, daß das in Straßburg 22 vorhandene Kopierdatum 1411, das im Basler Manuskript in 1442 verändert ist, sich nur auf den letzten eingetragenen Traktat bezogen habe, scheint die Vorlagefunktion von Straßburg 22 nicht auszuschließen. Angesichts der Herkunft des Fragments $\mathbf{C}$ aus dem Material des Basler Predigerklosters könnte auch eine Rolle gespielt haben, daß Löffler ebendort Predigermönch war, womit aber keineswegs behauptet werden soll, daß er an den Fragmenten $\mathbf{B}$ und $\mathbf{C}$ als Schreiber beteiligt gewesen sei. 
eben in Straßburg 22 ihre Konkordanzen besitzen ${ }^{20}$ - wenngleich vielleicht auch in nicht genau übereinstimmenden Fassungen.

Die Bedeutung der vier fraglichen Fragmente liegt zweifellos darin, daß sie für die Basler Region den Zeitraum zwischen dem späten 14. Jahrhundert und dem Basler Konzil mit greifbaren Zeugnissen einer mehrstimmigen geistlichen und weltlichen Musikübung belegen, einen Zeitraum also, den bisher nur jenes, freilich verlorene Straßburger Manuskript abdeckte: der Bestand an Quellen wird auf diese Weise bunter und vielfältiger. Für die Zeit nach etwa 1430 dürfte Basler Provenienz dann für Teile der Manuskripte Aosta und Trento 87 sowie für das Fragment Zwettl gelten. ${ }^{21}$ Wie auch immer: es beginnt sich die Ahnung an vorweisbaren Zeugnissen zu bestätigen, daß das oberrheinische Gebiet im 15. Jahrhundert musikalisch aktiver war, als es bisher allein das Manuskript Straßburg 22 bezeugt hat.

20 Konkordanzen von C mit Straßburg 22: Chanson Par maintes foys und Motette Exultet vena mea, diese der Straßburger Fassung erkennbar nahestehend; vgl. Lorenz Welker (wie oben, Anm. 11), Bd. 2, Inventar S. 26, Nr. 100/101, und S. 14, Nr. 41/42.

21 Vgl. dazu oben, Anm. 3; zur Handschrift Aosta sodann Marian Cobin, The Aosta Manuskript: A Central Source of Early-Fifteenth-Century Sacred Polyphony, Diss. (maschr.) New York University 1978, passim. 
